\title{
Compressed Sensing Image De-noising Algorithm Based on L1-L2 Norm Regularization
}

\author{
Liu Ziming ${ }^{1}$, Fang Changjie ${ }^{2+}$ \\ ${ }^{1}$ School of Automation, Chongqing University of Posts and Telecommunications, China \\ ${ }^{2}$ School of Science, Chongqing University of Posts and Telecommunications, China
}

\begin{abstract}
In this paper, we propose a compressed sensing image de-noising algorithm based on L1-L2 norm regularization. After the image is decomposed by the total variation spectral framework, L1 norm regularization is performed on the texture image, and L2 norm regularization is performed on the contour image, then the alternating direction method of multipliers (ADMM) is used for solution. The results of numerical experiment show that the proposed algorithm obtains higher peak signal-to-noise ratio (PSNR) and structural similarity (SSIM) than the compared compressed sensing algorithm and the total variation algorithm, and can effectively maintain the contour information and texture information of the image when de-noising.
\end{abstract}

Keywords: compressed sensing, regularization, ADMM, total variation.

\section{Introduction}

Image noise is a problem in the field of image processing, and the noisy image will bring great difficulties to the feature extraction of the in the later stage[1].Therefore, the effective image de-noising has been the focus of attention. The purpose of image de-noising is to recover unknown original picture from the noisy image .In 1992, Osher and Fatemi proposed a classical total variation de-noising model [2]. However, the traditional total variation algorithm will misjudge the noise as the edge of the image, leading to staircase effect, which makes the quality of de-noised image unsatisfactory. On the other hand, the improved compressed sensing algorithm[3] based on the traditional total variation model can quickly reconstruct and de-noise the image, but the texture and structural features of the image are not taken into account.

In order to overcome this difficulty, based on the compressed sensing algorithm of reference [4], we propose a compressed sensing de-noising algorithm based on L1-L2 regularization. First, the noisy image is decomposed by the spectral framework to obtain a contour image with a small amount of noise and a texture image with a lot of noise. Since the contour image is mainly a smooth region, and is low-frequency information; while the texture image mainly contains details and noise, and is a high-frequency region [5], therefore, inspired by the reference[6], different weighting methods are used for the contour image and the texture image according to the structural features of the image. The L2 weighting method is adopted for the contour image with a small amount of noise, and the L1 weighting is adopted for the texture image with a large amount of noise, which can help to avoid the staircase effect in de-noising. Then, by combining the two parts after decomposition with the compressed sensing algorithm, a complex model with two regular terms is obtained. In order to solve this complex model, the alternating direction method of multipliers [7] (ADMM) similar to that in reference [8] is used for iterative solution. However, different from reference [8], in this paper, the Barziilai-Borwein method [9] in the gradient descent algorithm is directly adopted to solve the first sub-problem of the ADMM algorithm. Finally, the experiment results demonstrate that the proposed

\footnotetext{
${ }^{+}$Corresponding author. Tel.: +86 13594142998
}

E-mail address: fangcj@cqupt.edu.cn 
method is effective, and the objective PSNR and SSIM are superior to those of other algorithms of compressed sensing reconstruction.

\section{Theoretical Foundation}

The classical total variation regularization model [2] is as follows:

$$
u=\arg \min _{u}\left(\frac{\kappa}{2} \int_{\Omega}|u-f|^{2} d u+\int_{\Omega}|D u| d u\right)
$$

where $u$ is the original image, $t$ represents noisy image, $D$ is the gradient operator of forward difference, $\Omega$ is the domain of the image, $\kappa$ is the parameter. The first term is a fitting term to ensure that the de-noised image is close to the original image; the second term is a regular term, which is also known as the total variation of $u$, and can promote the smoothness of the de-noised image.

The high-dimensional original value $u$ is recovered from the low-dimensional observed value $f=A u+b$, where $f \in R^{M}, u \in R^{N}, A \in R^{M^{*} N}(M \ll N)$ is the observation matrix. This kind of reconstruction problem is essentially an ill-posed inverse problem, which can be solved by optimization algorithm. Therefore, the total variation model combined with compressed sensing is as follows:

$$
u=\arg \min _{u}\left(\frac{\kappa}{2} \int_{\Omega}|A u-f|^{2} d u+\int_{\Omega}|D u| d u\right)
$$

The total variation spectral framework, which was proposed by Gilboa, can decompose the original image into contour image and texture image $[10,11]$. The image $u$ can be decomposed into two parts: contour $u_{L}$ and texture $u_{H}$.

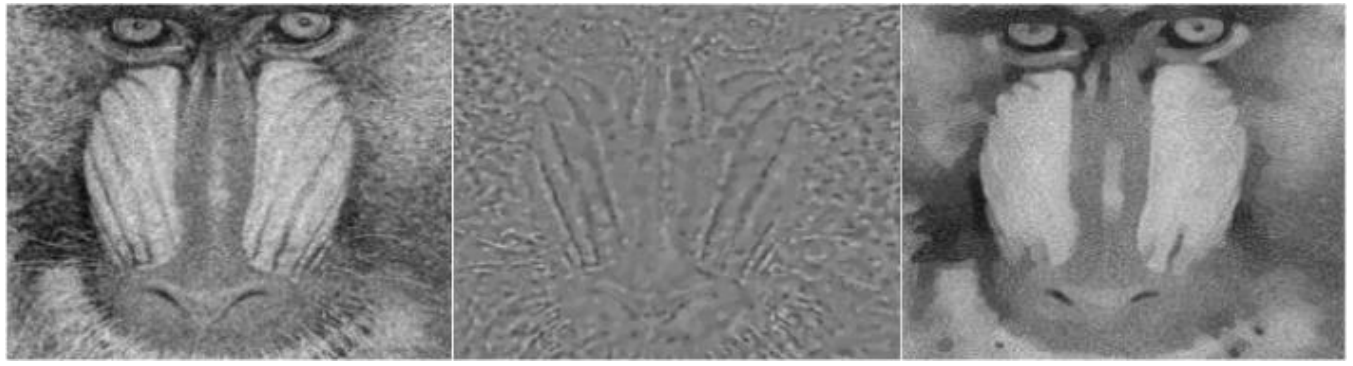

(a) original image (b) contour image (c) texture image

Fig. 1: Images obtained by the decomposition of noisy image

\section{Proposed Algorithm}

The compressed sensing de-noising algorithm based on L1-L2 norm regularization is improved based on the compressed sensing algorithm proposed in reference [4], and it combines the advantages of weighted regularization and compressed sensing. In order to maintain the texture and edge information of the image while avoiding the staircase effect, the decomposed images are weighted and regularized:

$$
\rho(u)=w_{H}\left\|D u_{H}\right\|_{1}+w_{L}\left\|D u_{L}\right\|_{2}
$$

where $w_{H}=\eta /(1+\eta), w_{L}=1 /(1+\eta), \eta$ is the absolute value of the image gradient mean.

The compressed sensing de-noising algorithm model based on L1-L2 norm regularization is:

$$
\left(u_{H}, u_{L}\right)=\arg \min _{\left(u_{H}, u_{L}\right)}\left(w_{H}\left\|D u_{H}\right\|_{1}+w_{L}\left\|D u_{L}\right\|_{2}+\frac{\kappa}{2}\|A u-f\|_{2}^{2}\right)
$$

The de-noising model (4) is a complex model with two regular terms. In order to facilitate the solution, ADMM is used to solve $u_{H}$ and $u_{L}$ respectively.

Fix $u_{L}$, the sub-problem of $u_{H}$ can be obtained:

$$
u_{H}=\arg \min _{u_{H}}\left(w_{H}\left\|D u_{H}\right\|_{1}+\frac{\mu_{H}}{2}\left\|A u_{H}-f_{H}\right\|_{2}^{2}\right)
$$

Fix $u_{H}$, the sub-problem of $u_{L}$ can be obtained: 


$$
u_{L}=\arg \min _{u_{L}}\left(w_{L}\left\|D u_{L}\right\|_{2}+\frac{\mu_{L}}{2}\left\|A u_{L}-f_{L}\right\|_{2}^{2}\right)
$$

where $f_{H}=f-A u_{L}, f_{L}=f-A u_{H}, \mu_{H}$ and $\mu_{L}$ are the parameters. Obviously the problem after decomposition is easier to be solved than the original problem (4).

It is difficult to solve the sub-problem (5) directly. Therefore, the variable splitting [12] can be used to introduce the auxiliary variables $u_{1}$ and $d_{H}$. Set $u_{1}=A u_{H}$ and $d_{H}=D u_{H}$. Then the sub-problem (5) can be converted to a constrained optimization problem.

$$
\begin{aligned}
& u_{H}=\arg \min _{u_{H}}\left(w_{H}\left\|D u_{H}\right\|_{1}+\frac{\mu_{H}}{2}\left\|A u_{H}-f_{1}\right\|_{2}^{2}\right) \\
& \text { s.t. } u_{1}=A u_{H} \quad d_{H}=D u_{H}
\end{aligned}
$$

The problem (7) can be converted to an unconstrained optimization problem using the Lagrange Multiplier Method, and its corresponding Lagrangian function is as follows:

$$
\begin{aligned}
& L_{1}\left(u_{H}, d_{H}, u_{1} ; \gamma_{H}, \delta_{H} ; \mu\right)=\left\|d_{H}\right\|_{1}+\frac{\mu_{H}}{2 w_{H}}\left\|u_{1}-f_{H}\right\|_{2}^{2}+\frac{\beta_{H 1}}{2}\left\|A u_{H}-u_{1}\right\|_{2}^{2} \\
& +\frac{\beta_{H 2}}{2}\left\|D u_{H}-d_{H}\right\|_{2}^{2}-\gamma_{H}^{T}\left(A u_{H}-u_{1}\right)-\delta_{H}^{T}\left(D u_{H}-d_{H}\right)
\end{aligned}
$$

where $\beta_{H 1}$ and $\beta_{H 2}$ are penalty term coefficients, $\gamma_{H}$ and $\delta_{H}$ are augmented Lagrangian coefficient matrix.

Since equation (8) contains three variables: $u_{H}, d_{H}$ and $u_{1}$, it is complicated to solve. The ADMM can be used again to decompose it into three sub-problems with only one variable. Given $u_{H}^{K}, d_{H}^{K}, u_{1}^{k}, \gamma_{H}^{k}$, $\delta_{H}^{k}$ and $\mu_{H}^{k}$,we obtain

$$
\left\{\begin{array}{r}
u_{H}^{k+1}=\arg \min _{u_{H}} L_{1}\left(u_{H}, d_{H}^{k}, u_{1}^{k} ; \gamma_{H}^{k}, \delta_{H}^{k} ; \mu_{H}^{k}\right) \\
d_{H}^{k+1}=\arg \min _{d_{H}} L_{1}\left(u_{H}^{k+1}, d, u_{1}^{k} ; \gamma_{H}^{k}, \delta_{H}^{k} ; \mu_{H}^{k}\right) \\
u_{1}^{k+1}=\arg \min _{u_{1}} L_{1}\left(u_{H}^{k+1}, d_{H}^{k+1}, u ; \gamma_{H}^{k}, \delta_{H}^{k} ; \mu_{H}^{k+1}\right) \\
\gamma_{H}^{k+1}=\gamma_{H}^{k}-\beta_{H 1}\left(u_{1}^{k+1}-A u_{H}^{k+1}\right) \\
\delta_{H}^{k+1}=\delta_{H}^{k}-\beta_{H 2}\left(d_{H}^{k+1}-D u_{H}^{k+1}\right)
\end{array}\right.
$$

1) Fix $d_{H}^{K}$ and $u_{1}^{k}$ to solve $u_{H}^{k+1}, u_{H}$ can be expressed as:

$$
\begin{array}{r}
u_{H}=\arg \min _{u_{H}}\left(\frac{\beta_{H 1}}{2}\left\|A u_{H}-u_{1}\right\|_{2}^{2}+\frac{\beta_{H 2}}{2}\left\|D u_{H}-d_{H}\right\|_{2}^{2}\right. \\
\left.-\gamma_{H}^{T}\left(A u_{H}-u_{1}\right)-\delta_{H}^{T}\left(D u_{H}-d_{H}\right)\right)
\end{array}
$$

Gradient descent can be adopted to solve $u_{H}, u_{H}^{k+1}=u_{H}^{k}-\alpha_{H}^{k} g_{H}^{k}$, where $g_{H}^{k}$ can be obtained by derivation, and $\alpha_{H}^{k}$ can be obtained by Barzilai-Borwein method [9].

$$
\begin{aligned}
& g_{H}^{k}=\beta_{H 1} A^{T}\left(A u_{H}-u_{1}^{k}\right)+\beta_{H 2} D^{T}\left(D u_{H}-d_{H}^{k}\right)-A^{T} \gamma_{H}^{k}-D^{T} \delta_{H}^{k} \\
& \alpha_{H}^{k}=\frac{v_{H}^{k T} v_{H}^{k}}{v_{H}^{k T} y_{H}^{k}}=\frac{\left(u_{H}^{k}-u_{H}^{k-1}\right)^{T}\left(u_{H}^{k}-u_{H}^{k-1}\right)}{\left(u_{H}^{k}-u_{H}^{k-1}\right)^{T}\left[g_{H}^{k}\left(u_{H}^{k}\right)-g_{H}^{k}\left(u_{H}^{k-1}\right)\right]}
\end{aligned}
$$

2)Fix $u_{H}^{k+1}$ and $u_{1}^{k}$ to solve $d_{H}^{k+1}, d_{H}$ can be described as :

$$
d_{H}=\arg \min _{d_{H}}\left(\left\|d_{H}\right\|_{1}+\left(\beta_{H 2} / 2\right)\left\|D u_{H}-d_{H}+\delta_{H}^{k} / \beta_{H 2}\right\|_{2}^{2}\right)
$$

Equation (12) can be solved by the Shrinkage method [13]:

$$
d_{H}^{k+1}=\max \left(\left\|D u_{H}^{k+1}+\delta_{H}^{k} / \beta_{H 2}\right\|_{1}-1 / \beta_{H 2}, 0\right) *\left(D u_{H}^{k+1}+\delta_{H}^{k} / \beta_{H 2}\right) /\left\|D u_{H}^{k+1}+\delta_{H}^{k} / \beta_{H 2}\right\|_{1}
$$

3)Fix $u_{H}^{k+1}$ and $d_{H}^{k+1}$ to solve $u_{1}^{k+1}, u_{1}$ can be expressed as: 


$$
\min _{u_{1}}\left[\left(\mu_{H} / 2 w_{H}\right)\left\|u_{1}-f_{H}\right\|_{2}^{2}+\left(\beta_{H 1} / 2\right)\left\|u_{1}-\left(A u_{H}+\gamma_{H} / \beta_{H 1}\right)\right\|_{2}^{2}\right]
$$

Set the derivation of equation (14) equals to zero, then

$$
u_{1}^{k+1}=\frac{\beta_{H 1}\left(A u_{H}^{k+1}+\frac{\gamma_{H}^{k+1}}{\beta_{H 1}}\right)+f_{H} \mu_{H}^{k+1} / w_{H}}{\beta_{H 1}+\mu_{H}^{k+1} / w_{H}}
$$

where the update of $\mu$ needs to meet the deviation criteria[14]. Set $\theta_{H}=\tau\left(\sigma_{H}^{k+1}\right)^{2} M N$, where $\sigma_{H}^{k+1}$ is the noise standard deviation of $f_{H}^{k}, \mathrm{MN}$ is the total number of pixels in the image. If $\left\|u_{1}^{k+1}-f_{H}^{k}\right\|<c_{H}^{k+1}$, then $\mu_{H}$ is approaching 0. If $\left\|u_{1}^{k+1}-f_{H}^{k}\right\|>c_{H}^{k+1}$, we set $c_{H}^{k+1}=\left\|u_{1}^{k+1}-f_{H}^{k}\right\|$ and hence we have:

$$
\mu_{H}^{k+1}=\left(\beta_{H 1} / \sqrt{c_{H}^{k+1}}\right)\left(\left\|A u_{H}^{k+1}+\gamma_{H}^{k+1} / \beta_{H 1}-f_{H}\right\|_{2}^{2}-1\right.
$$

Similarly, two auxiliary variables $u_{2}$ and $d_{L}$ are also needed to solve the sub-problem of $u_{L}$. Set $u_{2}=A u_{L}$ and $d_{L}=D u_{L}$. In solving the sub-problem of $u_{L}$, the methods for solving $u_{L}^{k+1}$ and $u_{2}^{k+1}$ are similar to those for solving $u_{H}^{k+1}$ and $u_{1}^{k+1}$. In other words, $u_{L}^{k+1}$ and $u_{2}^{k+1}$ can be obtained from $u_{H}^{k+1}$ and $u_{1}^{k+1}$ by replacing the corresponding subscript $H$ with $L$, respectively. At the same time, $d_{L}^{k+1}$ can be obtained in the following way different from $d_{H}^{k+1}$; see (17) below.Given $u_{L}^{k}, d_{L}^{k}, u_{2}^{k}, \gamma_{L}^{k}, \delta_{L}^{k}$ and $\mu_{L}^{k}$, and fix $u_{L}^{k+1}$ and $u_{2}^{k}, d_{L}^{k+1}$ can be expressed as :

$$
d_{L}^{k+1}=\max \left(\sqrt{S^{k+1} * \bar{S}^{k+1}}-\frac{1}{\beta_{L 2}}, 0\right) * \frac{1}{\sqrt{S^{k+1} * \bar{S}^{k+1}}-\frac{1}{\beta_{L 2}}}
$$

where $S^{k+1}=D u_{L}^{k+1}-\delta_{L}^{k} / \beta_{L 2}$, and $\bar{S}$ denotes the conjugate of $S$.

\section{Experimental Results and Conclusion}

Four classical digital images of $256 \times 256$ pixels: Lena, Cameraman, Barbara and Baboon are selected for the experiment. All the experiments were performed on MATLAB R2010b 7.11. The computer processor used in the experiment is Intel(R) i5-7300HQ, and RAM is 8.00GB. The noise is the Gaussian white noise with standard deviations of $0.05,0.1,0.2$, and 0.3 respectively. The maximum number of iteration is set to 500; the algorithm precision tol is set as $10^{-4}$, that is, the algorithm stops when $\left\|u^{k+1}-u^{k}\right\| /\left\|u^{k}\right\|<t o l$. The penalty term parameters are set based on the experimental experience as $\beta_{H 1}=30, \beta_{H 2}=2 * 10^{3}$, $\beta_{L 1}=80, \beta_{L 2}=5$. SNR and SSIM are used as evaluation indexes for image reconstruction. The larger the index values of the two evaluations, the better. The unit of PSNR is dB, and the range of SSIM is from 0 to 1.The contrast experiment algorithms used in this work are the TV algorithm in reference[2] and compressed sensing algorithm in reference[4]. The compressed sensing algorithm and the proposed algorithm all use discrete wavelet transform (DWT) to sparse the noisy image. Gaussian random matrix is used for measurement matrix $A$, and the sampling ratio is 0.4 .

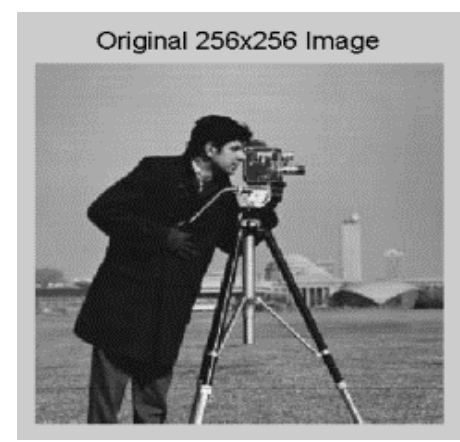

(a) original image

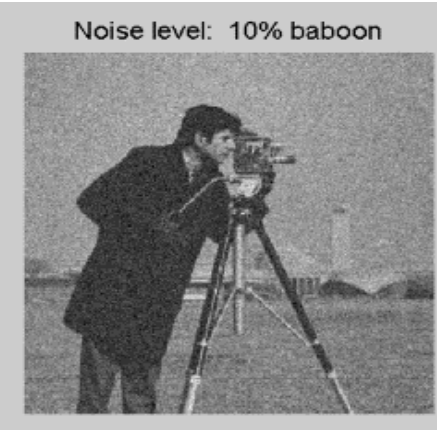

(b) noisy image

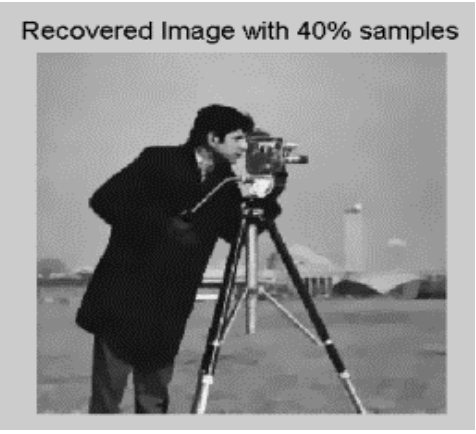

(c) recovered image

Fig. 2: The image of cameraman de-noised by the proposed algorithm when the noise is 0.1 . 


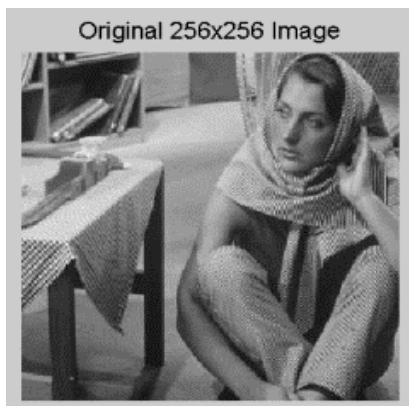

(a) original image

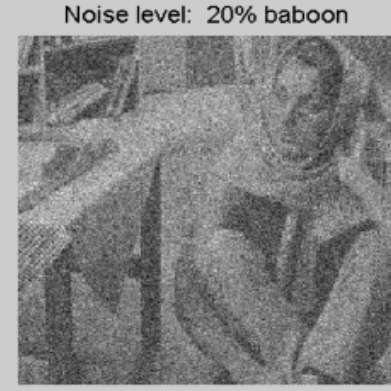

(b) noisy image

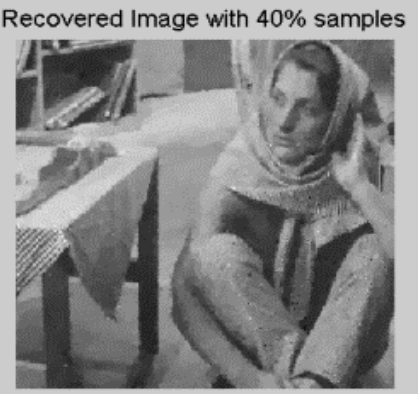

(c) recovered image

Fig. 3: The image of barbara de-noised by the proposed algorithm when the noise is 0.2 .

Table 1: Results of three de-noising algorithms

\begin{tabular}{|c|c|c|c|c|c|c|c|c|c|c|c|c|}
\hline & \multicolumn{6}{|c|}{ Lena } & \multicolumn{6}{|c|}{ Cameraman } \\
\hline & \multicolumn{2}{|c|}{ TV } & \multicolumn{2}{|c|}{ Reference[4] } & \multicolumn{2}{|c|}{$\begin{array}{l}\text { Proposed } \\
\text { algorithm }\end{array}$} & \multicolumn{2}{|c|}{$\mathrm{TV}$} & \multicolumn{2}{|c|}{ Reference[4] } & \multicolumn{2}{|c|}{$\begin{array}{l}\text { Proposed } \\
\text { algorithm }\end{array}$} \\
\hline Noise & PSNR & SSIM & PSNR & SSIM & PSNR & SSIM & PSNR & SSIM & PSNR & SSIM & PSNR & SSIM \\
\hline 0.05 & 30.56 & 0.69 & 31.81 & 0.70 & 32.94 & 0.72 & 26.27 & 0.69 & 27.39 & 0.69 & 28.5 & 0.72 \\
\hline 0.10 & 30.02 & 0.66 & 31.43 & 0.68 & 32.85 & 0.70 & 25.93 & 0.66 & 26.98 & 0.67 & 28.04 & 0.69 \\
\hline 0.20 & 28.34 & 0.62 & 30.65 & 0.67 & 32.50 & 0.68 & 24.75 & 0.55 & 26.52 & 0.63 & 28.01 & 0.65 \\
\hline \multirow[t]{3}{*}{0.30} & 26.45 & 0.51 & 29.35 & 0.55 & 31.28 & 0.63 & 23.27 & 0.45 & 25.64 & 0.57 & 27.57 & 0.63 \\
\hline & \multicolumn{6}{|c|}{ Barbara } & \multicolumn{6}{|c|}{ Baboon } \\
\hline & \multicolumn{2}{|c|}{ TV } & \multicolumn{2}{|c|}{ Reference[4] } & \multicolumn{2}{|c|}{ Proposed } & \multicolumn{2}{|c|}{ TV } & \multicolumn{2}{|c|}{ Reference[4] } & \multicolumn{2}{|c|}{ Proposed } \\
\hline Noise & PSNR & SSIM & PSNR & SSIM & PSNR & SSIM & PSNR & SSIM & PSNR & SSIM & PSNR & SSIM \\
\hline 0.05 & 23.65 & 0.61 & 26.51 & 0.68 & 27.96 & 0.70 & 27.75 & 0.65 & 29.96 & 0.69 & 31.84 & 0.79 \\
\hline 0.10 & 23.52 & 0.59 & 26.23 & 0.66 & 27.79 & 0.68 & 27.54 & 0.64 & 29.88 & 0.66 & 31.42 & 0.77 \\
\hline 0.20 & 22.99 & 0.57 & 25.84 & 0.61 & 27.66 & 0.66 & 26.80 & 0.62 & 29.31 & 0.62 & 30.73 & 0.71 \\
\hline 0.30 & 22.16 & 0.53 & 25.08 & 0.57 & 26.54 & 0.59 & 25.76 & 0.59 & 28.41 & 0.61 & 29.70 & 0.67 \\
\hline
\end{tabular}

It can be seen from Table 1 that for the de-noising effect of the four test images, the value of PSNR is more than $1 \mathrm{~dB}$ compared to the contrast experiment algorithms. Especially when the image noise is large, the SSIM value of the proposed algorithm is higher, indicating that the de-noised image has a good structural similarity with the original image. This is because different regularization is adopted for the texture and contour image according to the different features during the de-noising process, which allows better texture features of the image after de-noising.

The proposed algorithm is a compressed sensing de-noising algorithm based on the L1-L2 norm regularization. Firstly, the image is decomposed into a contour image and a texture image by the total variation spectral framework method, and then the contour image and the texture image are regularized according to the image structural features. Then, ADMM is adopted for solution. Finally, the experimental results demonstrate that the de-noising effect of the proposed algorithm is better than that of the contrast experiment algorithms. However, it is worth noting that the gradient descent algorithm used for solving subproblems has a large number of iterative steps. In future research, the conjugate gradient algorithm with fewer iteration steps can be considered.

\section{References}

[1] W. Wang, and C. J. He. A fast and effective algorithm for a poisson de-noising model with total variation. IEEE Signal Processing Letters. 2017, 24(3): 269-273.

[2] L. I. Rudin, S. Osher, and E. Fatemi. Nonlinear total variation based noise removal algorithms. Physical D. 1992, 60(1-4): 259-268.

[3] J. Wang, and C. L. Yang. An improved total variation model for image de-noising based on compression perception. Computer Digital Eng. 2007, 45(9): 1833-1836.(in Chinese)

[4] Z. P. Liu, and Y. Y. Chen. Total variation image de-noising algorithm base on compressed sensing. Test and Measurement Technology. 2018, 130(4): 53-58. (in Chinese) 
[5] X. Liu, and L. Huang. A new nonlocal total variation regularization algorithm for image de-noising. Mathematics Computers in Simulation.2014, 97, 224-233.

[6] L. H. Yu, G. D. Liu, and C. J. Lin. An adaptive total variation de-noising algorithm for printed circuit board images. Technology Eng. Sci. 2019, 19(19): 207-213. (in Chinese)

[7] J. Yang, Y. Zhang, and W. Yin. A fast alternating direction method for tv 11-12 signal reconstruction from partial fourier data. IEEE Journal of Selected Topics in Signal Processing. 2010, 4(2): 288-297.

[8] X. M. Yang. , Y. Q. Xiang, and Y. N. Liu. Image deblurring method with fraction-order total variation and adaptive regularization parameters. Advanced Eng. Sci. 2018, 50(6): 205-211. (in Chinese)

[9] J. Barzilai, and J. M. Borwein. Two-point step size gradient methods. IMA Journal of Numerical Analysis.1988, 8(1): 141-148.

[10] M. Chen, H. Zhang, and G. Lin, et al. A new local and nonlocal total variation regularization model for image denoising. Cluster Computing.2019, 22: 7611-7627.

[11] Gilboa G. A total variation spectral framework for scale and texture. Imaging Sci.2014, 7(4): 1937-1961.

[12] Z. Qin, D. Goldfarn, and S. Ma. An alternating direction method for total variation de-noising. Optimization Methods Software.2015, 30(3): 594-615.

[13] Y. Wang, J. Yang, and W. Yin. A new alternating minimization algorithm for total variation image reconstruction. International Conference on Wireless, Mobile and Multi-Media, 2015.

[14] V. A. Morozov. Methods for solving incorrectly posed problems. Springer-verlag Press, 1984. 